\title{
Inactivated Vaccine
}

National Cancer Institute

\section{Source}

National Cancer Institute. Inactivated Vaccine. NCI Thesaurus. Code C29694.

A preparation of killed microorg anisms that is intended to prevent or treat an infectious disease by inducing active immunity to the causative microorganism. 